\title{
Viscoelasticity of molecular rearrangements controls protein function
}

\author{
Nonlinearity of a Voltage-Gated Potassium Channel Revealed \\ by the Mechanical Susceptibility, by Amila Ariyaratne and Giovanni \\ Zocchi, Physical Review X 3, 011010 (2013).
}

Recommended with a commentary by Elisha Moses, Weizmann Institute

At which point does molecular behavior make the cross over to a bulk behavior? This question, usually asked in the context of transitions from the quantum to the classical or the thermodynamic regimes, is getting a new perspective from recent work of Zocchi and co-workers at UCLA. Their findings clearly indicate that a continuum approach can describe the mechanical response of some macromolecules, specifically that of proteins, the molecular machinery of living organisms.

The first observation of viscoelastic behavior in the internal dynamics of a molecule was enabled by an improvement of about two orders of magnitude in measuring the average deformation of an ensemble of molecules, resulting in sub angstrom resolution [1]. This allowed for a precise determination of the response of an enzyme, guanylate kinase, to the mechanical force exerted by an electrically driven gold nanoparticle that is tethered to it. Up to a strain $\varepsilon \sim 1 \%$ a linear elasticity regime was observed, which should be compared to a normal crystalline solid that crosses to the plastic regime for $\varepsilon \sim 0.1$. Beyond that, a viscoelastic regime exists, characterized by internal friction in the molecule [2]. Their most recent work shows that another protein of great importance, the potassium voltage gated channel KvAp, is also characterized by a viscoelastic transition. In this case the viscoelasticity is manifest as a phase shift that appears in the response to electric forcing of high enough frequency, as a function of amplitude [3].

The authors believe that the source of the viscoelasticity is in conformational changes that the macromolecules undergo, which is a crucial aspect of their functionality. They furthermore suggest that ligand induced conformational changes generally operate in such a viscoelastic regime. A simple model that incorporates an elastic term with a dissipative one describes the behavior of the molecules in a surprisingly 
good manner. However, this cannot explain the very large internal friction that is measured, nor does it account for the biologically relevant times scales at which the transition to elastic behavior occurs. Clearly, this system calls for a fundamental theory that will yield the viscoelastic features from basic considerations.

\section{References}

[1] Elasticity of Globular Proteins Measured from the ac Susceptibility, Yong Wang and Giovanni Zocchi, Phys. Rev. Lett. 105, 238104 (2010).

[2] Viscoelastic Transition and Yield Strain of the Folded Protein, Yong Wang and Giovanni Zocchi, PLoS ONE 6(12): e28097, (2011).

[3] Nonlinearity of a Voltage-Gated Potassium Channel Revealed by the Mechanical Susceptibility, Amila Ariyaratne and Giovanni Zocchi, Phys. Rev. X 3, 011010 (2013). 